\title{
PERTURBATIONS OF AN OSTROWSKI TYPE INEQUALITY AND APPLICATIONS
}

\author{
NENAD UJEVIĆ
}

Received 28 December 2001

\begin{abstract}
Two perturbations of an Ostrowski type inequality are established. New error bounds for the mid-point, trapezoid, and Simpson quadrature rules are derived. These error bounds can be much better than some recently obtained bounds. Applications in numerical integration are also given.
\end{abstract}

2000 Mathematics Subject Classification: 26D10, 41A55, 65D30.

1. Introduction. In the recent years a number of authors have written about generalizations of Ostrowski's inequality. For example, this topic is considered in $[1,4,5]$. In [4], Dragomir et al. proved the following generalization of Ostrowski's inequality.

THEOREM 1.1 (see [4]). Let $f:[a, b] \rightarrow \mathbb{R}$ be continuous on $[a, b]$, differentiable on $(a, b)$, and whose derivative $f^{\prime}:(a, b) \rightarrow \mathbb{R}$ is bounded on $(a, b)$. Denote $\left\|f^{\prime}\right\|_{\infty}=$ $\sup _{t \in[a, b]}\left|f^{\prime}(t)\right|<\infty$. Then

$$
\begin{aligned}
\mid \int_{a}^{b} f(t) d t & -\left[f(x)(1-\lambda)+\frac{f(a)+f(b)}{2} \lambda\right](b-a) \mid \\
& \leq\left[\frac{1}{4}(b-a)^{2}\left(\lambda^{2}+(1-\lambda)^{2}\right)+\left(x-\frac{a+b}{2}\right)^{2}\right]\left\|f^{\prime}\right\|_{\infty},
\end{aligned}
$$

for all $\lambda \in[0,1]$ and $a+\lambda((b-a) / 2) \leq x \leq b-\lambda((b-a) / 2)$.

Using (1.1), the authors obtained estimations of error for the mid-point, trapezoid, and Simpson quadrature formulae. They also gave applications of the mentioned results in numerical integration and for special means.

In this paper, we establish two perturbations of (1.1). Using the perturbations, we derive some new error bounds for the mid-point, trapezoid, and Simpson quadrature formulae. Similar perturbed inequalities are also considered in [2, 3]. We give applications in numerical integration. It is shown that these new bounds can give much better results than the bounds obtained in [4].

\section{Perturbed inequalities}

THEOREM 2.1. Let $I \subset \mathbb{R}$ be an open interval and $a, b \in I, a<b$. If $f: I \rightarrow \mathbb{R}$ is $a$ differentiable function such that $\gamma \leq f^{\prime}(t) \leq \Gamma$, for all $t \in[a, b]$, for some constants 
$\gamma, \Gamma \in \mathbb{R}$, then

$$
\begin{aligned}
& \left|(b-a)\left[\frac{\lambda}{2}(f(a)+f(b))+(1-\lambda) f(x)-\gamma(1-\lambda)\left(x-\frac{a+b}{2}\right)\right]-\int_{a}^{b} f(t) d t\right| \\
& \quad \leq(S-\gamma) \max \left\{\lambda \frac{b-a}{2}, x-a-\lambda \frac{b-a}{2}, b-x-\lambda \frac{b-a}{2}\right\}(b-a), \\
& \left|(b-a)\left[\frac{\lambda}{2}(f(a)+f(b))+(1-\lambda) f(x)-\Gamma(1-\lambda)\left(x-\frac{a+b}{2}\right)\right]-\int_{a}^{b} f(t) d t\right| \\
& \leq(\Gamma-S) \max \left\{\lambda \frac{b-a}{2}, x-a-\lambda \frac{b-a}{2}, b-x-\lambda \frac{b-a}{2}\right\}(b-a),
\end{aligned}
$$

where $S=(f(b)-f(a)) /(b-a)$ and $a+\lambda((b-a) / 2) \leq x \leq b-\lambda((b-a) / 2)$, for $\lambda \in[0,1]$.

Proof. Define the mapping

$$
K(x, t)= \begin{cases}t-\left(a+\lambda \frac{b-a}{2}\right), & t \in[a, x], \\ t-\left(b-\lambda \frac{b-a}{2}\right), & t \in(x, b] .\end{cases}
$$

Integrating by parts, we have

$$
\begin{aligned}
\int_{a}^{b} K(x, t) f^{\prime}(t) d t & =\int_{a}^{x}\left[t-\left(a+\lambda \frac{b-a}{2}\right)\right] f^{\prime}(t) d t+\int_{x}^{b}\left[t-\left(b-\lambda \frac{b-a}{2}\right)\right] f^{\prime}(t) d t \\
& =(b-a)\left[\frac{\lambda}{2}(f(a)+f(b))+(1-\lambda) f(x)\right]-\int_{a}^{b} f(t) d t .
\end{aligned}
$$

We also have

$$
\begin{aligned}
\int_{a}^{b} K(x, t) d t & =\int_{a}^{x}\left[t-\left(a+\lambda \frac{b-a}{2}\right)\right] d t+\int_{x}^{b}\left[t-\left(b-\lambda \frac{b-a}{2}\right)\right] d t \\
& =\frac{1}{2}\left[(x-a)-\lambda \frac{b-a}{2}\right]^{2}-\frac{1}{2}\left[(x-b)+\lambda \frac{b-a}{2}\right]^{2} \\
& =(1-\lambda)(b-a)\left(x-\frac{a+b}{2}\right) .
\end{aligned}
$$

Let $C \in \mathbb{R}$ be a constant. From (2.4) and (2.5) it follows that

$$
\begin{aligned}
\int_{a}^{b} K(x, t)\left[f^{\prime}(t)-C\right] d t= & \int_{a}^{b} K(x, t) f^{\prime}(t) d t-C \int_{a}^{b} K(x, t) d t \\
=(b-a)[ & \frac{\lambda}{2}(f(a)+f(b))+(1-\lambda) f(x) \\
& \left.\quad-C(1-\lambda)\left(x-\frac{a+b}{2}\right)\right]-\int_{a}^{b} f(t) d t .
\end{aligned}
$$


If we choose $C=\gamma$ in (2.6), then we get

$$
\begin{gathered}
(b-a)\left[\frac{\lambda}{2}(f(a)+f(b))+(1-\lambda) f(x)-\gamma(1-\lambda)\left(x-\frac{a+b}{2}\right)\right]-\int_{a}^{b} f(t) d t \\
=\int_{a}^{b} K(x, t)\left[f^{\prime}(t)-\gamma\right] d t .
\end{gathered}
$$

On the other hand, we have

$$
\begin{aligned}
\left|\int_{a}^{b} K(x, t)\left[f^{\prime}(t)-\gamma\right] d t\right| & \leq \max _{t \in[a, b]}|K(x, t)| \int_{a}^{b}\left|f^{\prime}(t)-\gamma\right| d t \\
\max _{t \in[a, b]}|K(x, t)| & =\max \left\{\lambda \frac{b-a}{2}, x-a-\lambda \frac{b-a}{2}, b-x-\lambda \frac{b-a}{2}\right\} .
\end{aligned}
$$

Since

$$
\int_{a}^{b}\left|f^{\prime}(t)-\gamma\right| d t=f(b)-f(a)-\gamma(b-a)=(S-\gamma)(b-a),
$$

from (2.7), (2.8), and (2.9) it follows that (2.1) holds.

If we choose $C=\Gamma$ in (2.6), then we get

$$
\begin{aligned}
& (b-a)\left[\frac{\lambda}{2}(f(a)+f(b))+(1-\lambda) f(x)-\Gamma(1-\lambda)\left(x-\frac{a+b}{2}\right)\right]-\int_{a}^{b} f(t) d t \\
& =\int_{a}^{b} K(x, t)\left[f^{\prime}(t)-\Gamma\right] d t \\
& \int_{a}^{b}\left|f^{\prime}(t)-\Gamma\right| d t=\Gamma(b-a)-(f(b)-f(a))=(\Gamma-S)(b-a) .
\end{aligned}
$$

From (2.11) and (2.9), we easily get (2.2).

COROLLARY 2.2. Under the assumptions of Theorem 2.1, we have

$$
\begin{aligned}
& \mid f(x)(b-a)-\gamma(b-a)\left(x-\frac{a+b}{2}\right)-\int_{a}^{b} f(t) d t \mid \\
& \leq(S-\gamma)\left[\frac{b-a}{2}+\left|x-\frac{a+b}{2}\right|\right](b-a), \\
&\left|f(x)(b-a)-\Gamma(b-a)\left(x-\frac{a+b}{2}\right)-\int_{a}^{b} f(t) d t\right| \\
& \leq(\Gamma-S)\left[\frac{b-a}{2}+\left|x-\frac{a+b}{2}\right|\right](b-a) .
\end{aligned}
$$

Proof. We set $\lambda=0$ in (2.1) and (2.2). Then we have

$$
\max \{x-a, b-x\}=\frac{1}{2}[b-a+|2 x-a-b|]=\frac{b-a}{2}+\left|x-\frac{a+b}{2}\right| .
$$

Now, it is not difficult to see that (2.12) and (2.13) are valid. 
REMARK 2.3. In the above proof, we used

$$
\max \{A, B\}=\frac{1}{2}[A+B+|A-B|], \quad A, B \in \mathbb{R} .
$$

If we set $x=(a+b) / 2$ in (2.12) and (2.13), then we get corresponding mid-point inequalities.

COROLLARY 2.4. Under the assumptions of Theorem 2.1, we have

$$
\begin{aligned}
& \left|\frac{b-a}{2}[f(a)+f(b)]-\int_{a}^{b} f(t) d t\right| \leq(S-\gamma) \frac{(b-a)^{2}}{2} \\
& \left|\frac{b-a}{2}[f(a)+f(b)]-\int_{a}^{b} f(t) d t\right| \leq(\Gamma-S) \frac{(b-a)^{2}}{2} .
\end{aligned}
$$

Proof. We set $\lambda=1$ in (2.1) and (2.2). Then we have $x=(a+b) / 2$ and

$$
\max \left\{\lambda \frac{b-a}{2}, x-a-\lambda \frac{b-a}{2}, b-x-\lambda \frac{b-a}{2}\right\}=\frac{b-a}{2} .
$$

The proof is now obvious.

COROLLARY 2.5. Under the assumptions of Theorem 2.1, we have

$$
\begin{array}{r}
\left|(b-a)\left[\frac{f(a)+f(b)}{4}+\frac{1}{2} f(x)-\frac{\gamma}{2}\left(x-\frac{a+b}{2}\right)\right]-\int_{a}^{b} f(t) d t\right| \\
\leq(S-\gamma)\left[\frac{b-a}{4}+\left|x-\frac{a+b}{2}\right|\right](b-a), \\
\left|(b-a)\left[\frac{f(a)+f(b)}{4}+\frac{1}{2} f(x)-\frac{\Gamma}{2}\left(x-\frac{a+b}{2}\right)\right]-\int_{a}^{b} f(t) d t\right| \\
\leq(\Gamma-S)\left[\frac{b-a}{4}+\left|x-\frac{a+b}{2}\right|\right](b-a) .
\end{array}
$$

Proof. We set $\lambda=1 / 2$ in (2.1) and (2.2). Then we have

$$
\begin{aligned}
\max & \left\{\frac{b-a}{4}, x-\frac{3 a+b}{4}, \frac{a+3 b}{4}-x\right\} \\
& =\max \left\{\frac{1}{2}\left(x-a+\left|x-\frac{a+b}{2}\right|\right), \frac{1}{2}\left(b-x+\left|x-\frac{a+b}{2}\right|\right)\right\} \\
& =\frac{1}{4}\left[b-a+2\left|x-\frac{a+b}{2}\right|+|2 x-(a+b)|\right]=\frac{b-a}{4}+\left|x-\frac{a+b}{2}\right| .
\end{aligned}
$$

The proof of (2.19) and (2.20) is now obvious.

REMARK 2.6. If we set $x=(a+b) / 2$ in (2.19) and (2.20), then we get corresponding inequalities which do not depend on $x$. 
COROLLARY 2.7. Under the assumptions of Theorem 2.1, we have

$$
\begin{aligned}
\mid \frac{b-a}{6}[f(a)+4 f(x)+f(b)] & -\frac{2 \gamma}{3}\left(x-\frac{a+b}{2}\right)-\int_{a}^{b} f(t) d t \mid \\
& \leq(S-\gamma)\left[\frac{b-a}{3}+\left|x-\frac{a+b}{2}\right|\right](b-a), \\
\mid \frac{b-a}{6}[f(a)+4 f(x)+f(b)] & -\frac{2 \Gamma}{3}\left(x-\frac{a+b}{2}\right)-\int_{a}^{b} f(t) d t \mid \\
& \leq(\Gamma-S)\left[\frac{b-a}{3}+\left|x-\frac{a+b}{2}\right|\right](b-a) .
\end{aligned}
$$

Proof. We set $\lambda=1 / 3$ in (2.1) and (2.2). Then we have

$$
\begin{aligned}
\max \{\lambda & \left.\frac{b-a}{2}, x-a-\lambda \frac{b-a}{2}, b-x-\lambda \frac{b-a}{2}\right\} \\
& =\max \left\{\frac{b-a}{6}, x-\frac{5 a+b}{6}, \frac{a+5 b}{6}-x\right\} \\
& =\max \left\{\frac{1}{2}\left(x-a+\left|x-\frac{2 a+b}{3}\right|\right), \frac{1}{2}\left(b-x+\left|x-\frac{a+2 b}{3}\right|\right)\right\} \\
& =\max \left\{\frac{b-a}{6}, \frac{b-a}{3}+\left|x-\frac{a+b}{2}\right|\right\} \\
& =\frac{1}{2}\left[\frac{b-a}{2}+\left|x-\frac{a+b}{2}\right|+\left|\frac{b-a}{6}+\left(x-\frac{a+b}{2}\right)\right|\right] \\
& =\frac{b-a}{3}+\left|x-\frac{a+b}{2}\right| .
\end{aligned}
$$

Now, it is not difficult to obtain the proof of inequalities (2.22) and (2.23).

REMARK 2.8. If we set $x=(a+b) / 2$ in (2.22) and (2.23), then we get corresponding Simpson's inequalities.

3. Applications in numerical integration. The next approximations of the integral $\int_{a}^{b} f(t) d t$ hold.

THEOREM 3.1. Let all assumptions of Theorem 2.1 hold. If $I_{n}=\left\{a=x_{0}<x_{1}<\right.$ $\left.\cdots<x_{n}=b\right\}$ is a given subdivision of the interval $[a, b]$ and $h_{i}=x_{i+1}-x_{i}, i=$ $0,1,2, \ldots, n-1$, then

$$
\int_{a}^{b} f(t) d t=A\left(I_{n}, \xi, f\right)+\mathbb{R}_{\gamma}\left(I_{n}, \xi, f\right)
$$

where

$$
A\left(I_{n}, \xi, f\right)=\sum_{i=0}^{n-1}\left[f\left(\xi_{i}\right)-\gamma\left(\xi_{i}-\frac{x_{i}+x_{i+1}}{2}\right)\right] h_{i},
$$


for $x_{i} \leq \xi_{i} \leq x_{i+1}, i=0,1,2, \ldots, n-1$. The remainder term satisfies

$$
\left|\mathbb{R}_{\gamma}\left(I_{n}, \xi, f\right)\right| \leq \sum_{i=0}^{n-1}\left(S_{i}-\gamma\right)\left[\frac{h_{i}}{2}+\left|\xi_{i}-\frac{x_{i}+x_{i+1}}{2}\right|\right] h_{i},
$$

where $S_{i}=\left(f\left(x_{i+1}\right)-f\left(x_{i}\right)\right) / h_{i}, i=0,1, \ldots, n-1$. Also,

$$
\int_{a}^{b} f(t) d t=A\left(I_{n}, \xi, f\right)+\mathbb{R}_{\Gamma}\left(I_{n}, \xi, f\right),
$$

where

$$
\left|\mathbb{R}_{\Gamma}\left(I_{n}, \xi, f\right)\right| \leq \sum_{i=0}^{n-1}\left(\Gamma-S_{i}\right)\left[\frac{h_{i}}{2}+\left|\xi_{i}-\frac{x_{i}+x_{i+1}}{2}\right|\right] h_{i} .
$$

Proof. We apply (2.12) to the interval $\left[x_{i}, x_{i+1}\right]$, then

$$
\begin{aligned}
\mid f\left(\xi_{i}\right) h_{i}-\gamma\left(\xi_{i}-\right. & \left.\frac{x_{i}+x_{i+1}}{2}\right) h_{i}-\int_{x_{i}}^{x_{i+1}} f(t) d t \mid \\
\leq & \left(S_{i}-\gamma\right)\left[\frac{h_{i}}{2}+\left|\xi_{i}-\frac{x_{i}+x_{i+1}}{2}\right|\right] h_{i},
\end{aligned}
$$

for $i=0,1, \ldots, n-1$. We also have

$$
f\left(\xi_{i}\right) h_{i}-\gamma\left(\xi_{i}-\frac{x_{i}+x_{i+1}}{2}\right) h_{i}-\int_{x_{i}}^{x_{i+1}} f(t) d t=\int_{x_{i}}^{x_{i+1}} \hat{K}\left(\xi_{i}, t\right)\left[f^{\prime}(t)-\gamma\right] d t
$$

where

$$
\hat{K}\left(\xi_{i}, t\right)= \begin{cases}t-x_{i}, & t \in\left[x_{i}, \xi_{i}\right], \\ t-x_{i+1}, & t \in\left(\xi_{i}, x_{i+1}\right],\end{cases}
$$

for $i=0,1, \ldots, n-1$. If we now sum (3.7) over $i$ from 0 to $n-1$ and apply the triangle inequality and (3.6), then we get (3.1), (3.2), and (3.3). In a similar way, we can prove that (3.4) and (3.5) hold.

REMARK 3.2. If we set $\xi_{i}=\left(x_{i}+x_{i+1}\right) / 2$ in Theorem 3.1, then we get the composite mid-point rule.

THEOREM 3.3. Let all the assumptions of Theorem 2.1 hold. If $I_{n}=\left\{a=x_{0}<x_{1}<\right.$ $\left.\cdots<x_{n}=b\right\}$ is a given subdivision of the interval $[a, b]$ and $h_{i}=x_{i+1}-x_{i}, i=$ $0,1,2, \ldots, n-1$, then

$$
\int_{a}^{b} f(t) d t=A_{T}\left(I_{n}, f\right)+\mathbb{R}_{T \gamma}\left(I_{n}, f\right),
$$


where

$$
\begin{gathered}
A_{T}\left(I_{n}, f\right)=\sum_{i=0}^{n-1} \frac{f\left(x_{i}\right)+f\left(x_{i+1}\right)}{2} h_{i}, \\
\left|\mathbb{R}_{T \gamma}\left(I_{n}, f\right)\right| \leq \frac{1}{2} \sum_{i=0}^{n-1}\left(S_{i}-\gamma\right) h_{i}^{2},
\end{gathered}
$$

and $S_{i}=\left(f\left(x_{i+1}\right)-f\left(x_{i}\right)\right) / h_{i}, i=0,1, \ldots, n-1$. Also

$$
\int_{a}^{b} f(t) d t=A_{T}\left(I_{n}, f\right)+\mathbb{R}_{T \Gamma}\left(I_{n}, f\right),
$$

where

$$
\left|\mathbb{R}_{T \Gamma}\left(I_{n}, f\right)\right| \leq \frac{1}{2} \sum_{i=0}^{n-1}\left(\Gamma-S_{i}\right) h_{i}^{2}
$$

Proof. We apply (2.16) to the interval $\left[x_{i}, x_{i+1}\right]$, then

$$
\left|\frac{f\left(x_{i}\right)+f\left(x_{i+1}\right)}{2} h_{i}-\int_{x_{i}}^{x_{i+1}} f(t) d t\right| \leq \frac{1}{2}\left(S_{i}-\gamma\right) h_{i}^{2},
$$

for $i=0,1, \ldots, n-1$. We also have

$$
\frac{f\left(x_{i}\right)+f\left(x_{i+1}\right)}{2} h_{i}-\int_{x_{i}}^{x_{i+1}} f(t) d t=\int_{x_{i}}^{x_{i+1}} \hat{K}_{i}(t)\left[f^{\prime}(t)-\gamma\right] d t,
$$

where

$$
\hat{K}_{i}(t)=t-\frac{x_{i}+x_{i+1}}{2} .
$$

If we now sum (3.15) over $i$ from 0 to $n-1$ and apply the triangle inequality and (3.14), then we get (3.9), (3.10), and (3.11). In a similar way, we can prove that (3.12) and (3.13) hold.

THEOREM 3.4. Let the assumptions of Theorem 3.1 hold. Then

$$
\int_{a}^{b} f(t) d t=A_{C}\left(I_{n}, \xi, f\right)+\mathbb{R}_{C \gamma}\left(I_{n}, \xi, f\right),
$$

where

$$
\begin{aligned}
A_{C}\left(I_{n}, \xi, f\right)= & \frac{1}{2} \sum_{i=0}^{n-1} \frac{f\left(x_{i}\right)+f\left(x_{i+1}\right)}{2} h_{i} \\
& +\frac{1}{2} \sum_{i=0}^{n-1} f\left(\xi_{i}\right) h_{i}-C \sum_{i=0}^{n-1}\left(\xi_{i}-\frac{x_{i}+x_{i+1}}{2}\right) h_{i},
\end{aligned}
$$

for $C=\gamma / 2$, and

$$
\left|\mathbb{R}_{C \gamma}\left(I_{n}, \xi, f\right)\right| \leq \sum_{i=0}^{n-1}\left(S_{i}-\gamma\right)\left[\frac{h_{i}}{4}+\left|\xi_{i}-\frac{x_{i}+x_{i+1}}{2}\right|\right] h_{i} .
$$


Also

$$
\int_{a}^{b} f(t) d t=A_{C}\left(I_{n}, \xi, f\right)+\mathbb{R}_{C \Gamma}\left(I_{n}, \xi, f\right)
$$

where

$$
\left|\mathbb{R}_{C \Gamma}\left(I_{n}, \xi, f\right)\right| \leq \sum_{i=0}^{n-1}\left(\Gamma-S_{i}\right)\left[\frac{h_{i}}{4}+\left|\xi_{i}-\frac{x_{i}+x_{i+1}}{2}\right|\right] h_{i}
$$

and $C=\Gamma / 2$.

Proof. We apply (2.19) to the interval $\left[x_{i}, x_{i+1}\right]$, then

$$
\begin{aligned}
\left|\frac{f\left(x_{i}\right)+f\left(x_{i+1}\right)}{4} h_{i}+\frac{1}{2} f\left(\xi_{i}\right) h_{i}-\frac{\gamma}{2}\left(\xi_{i}-\frac{x_{i}+x_{i+1}}{2}\right) h_{i}-\int_{x_{i}}^{x_{i+1}} f(t) d t\right| \\
\leq\left(S_{i}-\gamma\right)\left[\frac{h_{i}}{4}+\left|\xi_{i}-\frac{x_{i}+x_{i+1}}{2}\right|\right] h_{i},
\end{aligned}
$$

for $i=0,1, \ldots, n-1$. We also have

$$
\begin{array}{r}
\frac{f\left(x_{i}\right)+f\left(x_{i+1}\right)}{4} h_{i}+\frac{1}{2} f\left(\xi_{i}\right) h_{i}-\frac{\gamma}{2}\left(\xi_{i}-\frac{x_{i}+x_{i+1}}{2}\right) h_{i}-\int_{x_{i}}^{x_{i+1}} f(t) d t \\
=\int_{x_{i}}^{x_{i+1}} K\left(\xi_{i}, t\right)\left[f^{\prime}(t)-\gamma\right] d t,
\end{array}
$$

where

$$
K\left(\xi_{i}, t\right)= \begin{cases}t-\frac{3 x_{i}+x_{i+1}}{4}, & t \in\left[x_{i}, \xi_{i}\right], \\ t-\frac{x_{i}+3 x_{i+1}}{4}, & t \in\left(\xi_{i}, x_{i+1}\right],\end{cases}
$$

for $i=0,1, \ldots, n-1$.

If we now sum (3.23) over $i$ from 0 to $n-1$ and apply the triangle inequality and (3.22), then we get (3.17), (3.18), and (3.19). In a similar way we can prove that (3.20) and (3.21) hold.

REMARK 3.5. If we set $\xi_{i}=\left(x_{i}+x_{i+1}\right) / 2$ in Theorem 3.4, then we get corresponding composite rules which do not depend on $\xi$.

THEOREM 3.6. Let the assumptions of Theorem 3.1 hold. Then

$$
\int_{a}^{b} f(t) d t=A_{S}\left(I_{n}, \xi, f\right)+\mathbb{R}_{S \gamma}\left(I_{n}, \xi, f\right),
$$

where

$$
A_{S}\left(I_{n}, \xi, f\right)=\frac{1}{6} \sum_{i=0}^{n-1}\left[f\left(x_{i}\right)+4 f\left(\xi_{i}\right)+f\left(x_{i+1}\right)\right] h_{i}-S \sum_{i=0}^{n-1}\left(\xi_{i}-\frac{x_{i}+x_{i+1}}{2}\right) h_{i},
$$


for $S=2 \gamma / 3$, and

$$
\left|\mathbb{R}_{S \gamma}\left(I_{n}, \xi, f\right)\right| \leq \sum_{i=0}^{n-1}\left(S_{i}-\gamma\right)\left[\frac{h_{i}}{3}+\left|\xi_{i}-\frac{x_{i}+x_{i+1}}{2}\right|\right] h_{i} .
$$

Also

$$
\int_{a}^{b} f(t) d t=A_{S}\left(I_{n}, \xi, f\right)+\mathbb{R}_{S \Gamma}\left(I_{n}, \xi, f\right),
$$

where

$$
\left|\mathbb{R}_{S \Gamma}\left(I_{n}, \xi, f\right)\right| \leq \sum_{i=0}^{n-1}\left(\Gamma-S_{i}\right)\left[\frac{h_{i}}{3}+\left|\xi_{i}-\frac{x_{i}+x_{i+1}}{2}\right|\right] h_{i}
$$

and $S=2 \Gamma / 3$.

Proof. We apply (2.22) to the interval $\left[x_{i}, x_{i+1}\right]$, then

$$
\begin{aligned}
\mid \frac{1}{6}\left[f\left(x_{i}\right)+4 f\left(\xi_{i}\right)+f\left(x_{i+1}\right)\right] h_{i}-\frac{2 \gamma}{3} & \left(\xi_{i}-\frac{x_{i}+x_{i+1}}{2}\right) h_{i}-\int_{x_{i}}^{x_{i+1}} f(t) d t \mid \\
& \leq\left(S_{i}-\gamma\right)\left[\frac{h_{i}}{3}+\left|\xi_{i}-\frac{x_{i}+x_{i+1}}{2}\right|\right] h_{i},
\end{aligned}
$$

for $i=0,1, \ldots, n-1$. We also have

$$
\begin{array}{r}
\frac{1}{6}\left[f\left(x_{i}\right)+4 f\left(\xi_{i}\right)+f\left(x_{i+1}\right)\right] h_{i}-\frac{2 \gamma}{3}\left(\xi_{i}-\frac{x_{i}+x_{i+1}}{2}\right) h_{i}-\int_{x_{i}}^{x_{i+1}} f(t) d t \\
=\int_{x_{i}}^{x_{i+1}} K\left(\xi_{i}, t\right)\left[f^{\prime}(t)-\gamma\right] d t
\end{array}
$$

where

$$
K\left(\xi_{i}, t\right)= \begin{cases}t-\frac{5 x_{i}+x_{i+1}}{6}, & t \in\left[x_{i}, \xi_{i}\right], \\ t-\frac{x_{i}+5 x_{i+1}}{6}, & t \in\left(\xi_{i}, x_{i+1}\right],\end{cases}
$$

for $i=0,1, \ldots, n-1$.

If we now sum (3.31) over $i$ from 0 to $n-1$ and apply the triangle inequality and (3.30), then we get (3.25), (3.26), and (3.27). In a similar way we can prove that (3.28) and (3.29) hold.

REMARK 3.7. If we set $\xi_{i}=\left(x_{i}+x_{i+1}\right) / 2$ in Theorem 3.6, then we get the composite Simpson's rule.

The results obtained in this paper can be much better than the results obtained in [4]. We illustrate this fact for the composite trapezoid quadrature rule.

In [4], we can find the following result:

$$
\int_{a}^{b} f(t) d t=A_{T}\left(I_{n}, f\right)+\mathbb{R}_{T}\left(I_{n}, f\right),
$$


where

$$
\begin{gathered}
A_{T}\left(I_{n}, f\right)=\sum_{i=0}^{n-1} \frac{f\left(x_{i}\right)+f\left(x_{i+1}\right)}{2} h_{i}, \\
\left|\mathbb{R}_{T}\left(I_{n}, f\right)\right| \leq \frac{\left\|f^{\prime}\right\|_{\infty}}{4} \sum_{i=0}^{n-1} h_{i}^{2} .
\end{gathered}
$$

EXAMPLE 3.8. Choose $a=0, b>0, h_{i}=h=(b-a) / n, i=0,1, \ldots, n-1$, and $f(t)=$ $t^{k}, k>2$. Then

$$
f^{\prime}(t)=k t^{k-1}, \quad \gamma=0, \quad\left\|f^{\prime}\right\|_{\infty}=k b^{k-1}, \quad k>2 .
$$

From (3.11), we get the right-hand side,

$$
\frac{1}{2} \sum_{i=0}^{n-1}\left(S_{i}-\gamma\right) h_{i}^{2}=\frac{b^{k+1}}{2 n}, \quad k>2 .
$$

From (3.35), we get the right-hand side,

$$
\frac{\left\|f^{\prime}\right\|_{\infty}}{4} \sum_{i=0}^{n-1} h_{i}^{2}=\frac{k b^{k+1}}{4 n}, \quad k>2 .
$$

From (3.37) and (3.38) we see that, for this example, estimation (3.11) is better than estimation (3.35). In fact, if $k \gg 2$ then (3.11) is much better than (3.35).

REMARK 3.9. In similar ways we can show that estimations for the mid-point and Simpson's composite rules (see Remarks 3.2 and 3.7) can be much better than corresponding estimations obtained in [4].

\section{REFERENCES}

[1] G. A. Anastassiou, Ostrowski type inequalities, Proc. Amer. Math. Soc. 123 (1995), no. 12, 3775-3781.

[2] P. Cerone and S. S. Dragomir, Midpoint-type rules from an inequalities point of view, Handbook of Analytic-Computational Methods in Applied Mathematics (G. Anastassiou, ed.), Chapman \& Hall/CRC, Florida, 2000, pp. 135-200.

[3] _ , Trapezoidal-type rules from an inequalities point of view, Handbook of AnalyticComputational Methods in Applied Mathematics (G. Anastassiou, ed.), Chapman \& Hall/CRC, Florida, 2000, pp. 65-134.

[4] S. S. Dragomir, P. Cerone, and J. Roumeliotis, A new generalization of Ostrowski's integral inequality for mappings whose derivatives are bounded and applications in numerical integration and for special means, Appl. Math. Lett. 13 (2000), no. 1, 19-25.

[5] C. E. M. Pearce, J. Pečarić, N. Ujević, and S. Varošanec, Generalizations of some inequalities of Ostrowski-Grüss type, Math. Inequal. Appl. 3 (2000), no. 1, 25-34.

Nenad UjeVić: DePartment of Mathematics, University OF SPlit, TeSlina 12/III, 21000 SPLIT, CROATIA

E-mail address: ujevic@pmfst.hr 


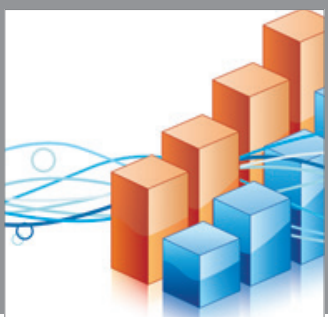

Advances in

Operations Research

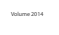

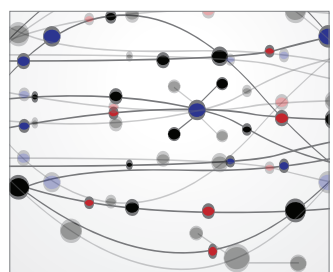

\section{The Scientific} World Journal
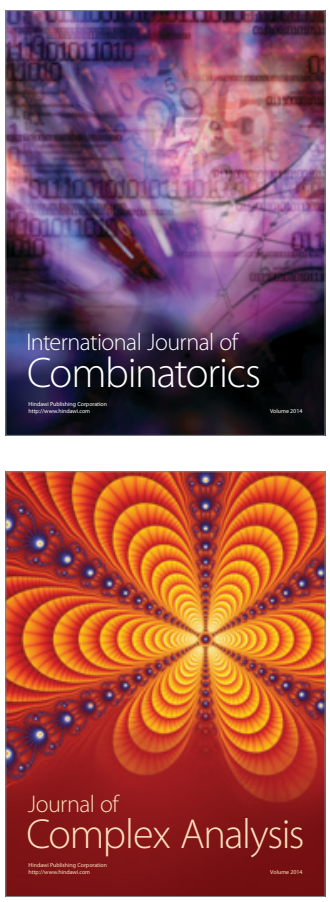

International Journal of

Mathematics and

Mathematical

Sciences
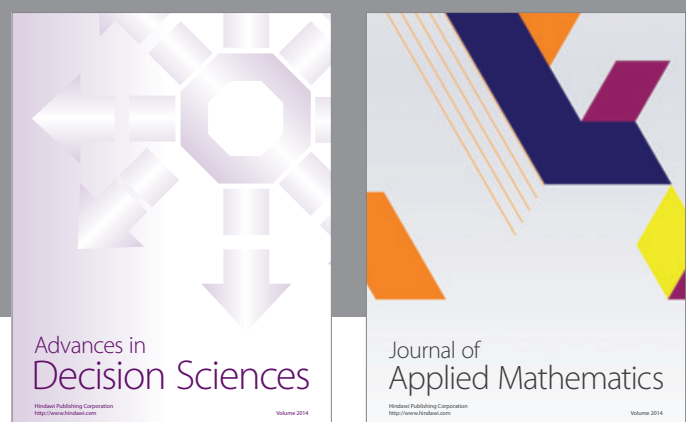

Journal of

Applied Mathematics
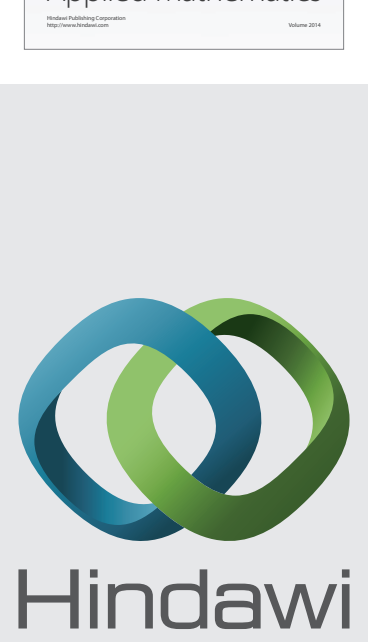

Submit your manuscripts at http://www.hindawi.com
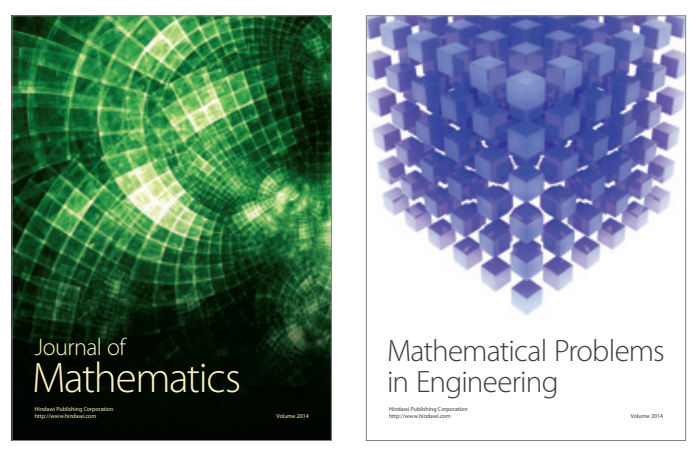

Mathematical Problems in Engineering
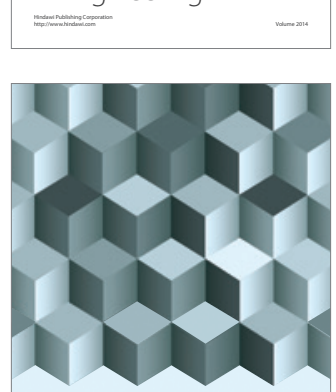

Journal of

Function Spaces
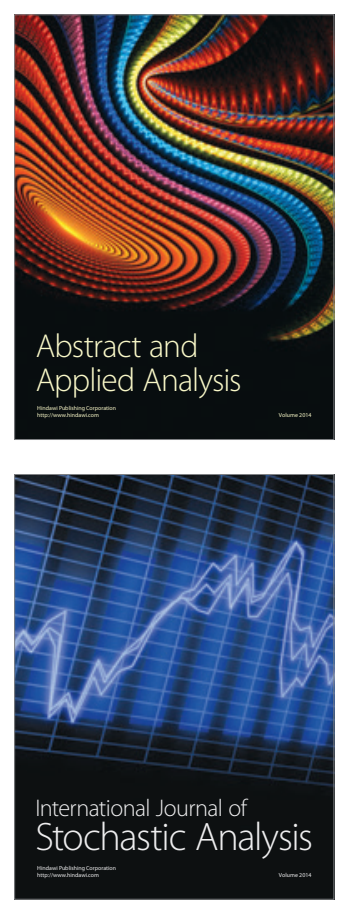

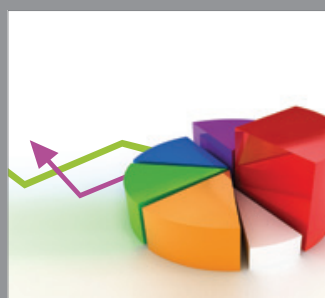

ournal of

Probability and Statistics

Promensencen
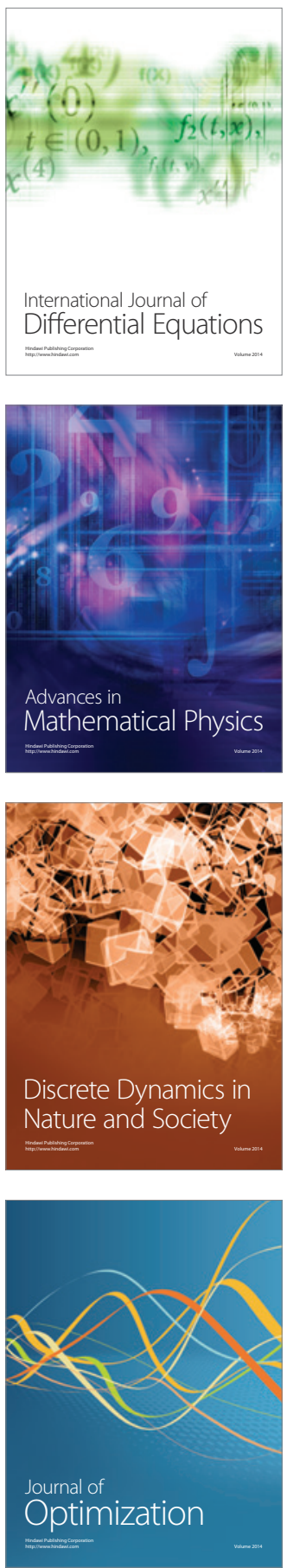